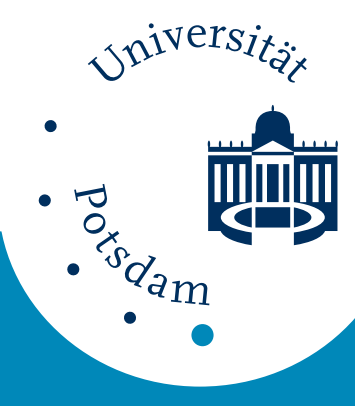

Universität Potsdam

Michael Beck, Niko Hildebrandt,

Hans-Gerd Löhmannsröben

\title{
Quantum Dots as Acceptors in FRET-Assays Containing Serum
}

first published in:

Biophotonics and New Therapy Frontiers / Romualda Grzymala, Olivier Haeberle (eds.). - S. 234 - 241. - (Proceedings of SPIE ; 6191)

ISBN: 9780819462473

doi: $10.1117 / 12.662722$

Postprint published at the institutional repository of Potsdam University: In: Postprints der Universität Potsdam :

Mathematisch-Naturwissenschaftliche Reihe ; 19

http://opus.kobv.de/ubp/volltexte/2006/950/

http://nbn-resolving.de/urn:nbn:de:kobv:517-opus-9504

Postprints der Universität Potsdam

Mathematisch-Naturwissenschaftliche Reihe ; 19 


\title{
Quantum Dots as Acceptors in FRET-Assays Containing Serum*
}

\author{
Michael Beck, Niko Hildebrandt, Hans-Gerd Löhmannsröben \\ Physikalische Chemie, Institut für Chemie und Interdisziplinäres Zentrum für Photonik, Universität \\ Potsdam, Karl-Liebknecht-Str. 24-25, 14476 Potsdam-Golm (Germany), \\ Tel.: ++49 (0) 331 / 977 5218, Fax: ++49 (0) 331 / 977 5058, e-mail: mbeck@chem.uni-potsdam.de
}

\begin{abstract}
Quantum dots (QDs) are common as luminescing markers for imaging in biological applications because their optical properties seem to be inert against their surrounding solvent. This, together with broad and strong absorption bands and intense, sharp tuneable luminescence bands, makes them interesting candidates for methods utilizing Förster Resonance Energy Transfer (FRET), e. g. for sensitive homogeneous fluoroimmunoassays (FIA). In this work we demonstrate energy transfer from $\mathrm{Eu}^{3+}$-trisbipyridin (Eu-TBP) donors to CdSe-ZnS-QD acceptors in solutions with and without serum. The QDs are commercially available CdSe-ZnS core-shell particles emitting at $655 \mathrm{~nm}$ (QD655). The FRET system was achieved by the binding of the streptavidin conjugated donors with the biotin conjugated acceptors. After excitation of Eu-TBP and as result of the energy transfer, the luminescence of the QD655 acceptors also showed lengthened decay times like the donors. The energy transfer efficiency, as calculated from the decay times of the bound and the unbound components, amounted to $37 \%$. The Förster-radius, estimated from the absorption and emission bands, was ca. $77 \AA$. The effective binding ratio, which not only depends on the ratio of binding pairs but also on unspecific binding, was obtained from the donor emission dependent on the concentration. As serum promotes unspecific binding, the overall FRET efficiency of the assay was reduced. We conclude that QDs are good substitutes for acceptors in FRET if combined with slow decay donors like Europium. The investigation of the influence of the serum provides guidance towards improving binding properties of QD assays.
\end{abstract}

Keywords: Quantum Dot, Luminescence, Serum, Europium, Immunoassay, Energy Transfer, FRET

\section{INTRODUCTION}

Quantum dots (QDs) are gaining more and more interest for biological applications. In that context they are chiefly used as luminescing markers since their optical properties seem to be inert against their surrounding solvent. This, together with broad and strong absorption bands and intense, sharp tuneable and luminescence bands, makes them interesting candidates for Förster Resonance Energy Transfer (FRET). The use of QDs as donors in FRET has already been reported, ${ }^{1 \text { a-d }}$ whereas their employment as acceptors has proven to be difficult, ${ }^{2}$ and was only recently unequivocally demonstrated. ${ }^{3}$ The FRET technique is useful for diagnostic methods like sensitive homogeneous fluoroimmunoanalysis $(\text { FIA })^{\dagger}$. With their unique and robust photophysical properties, QDs are suitable for FIA optimization and offer the potential for multiplexing. (For further description of immunoanalysis techniques see Ref.4.)

FIA is a diagnostic tool for detecting small amounts of specific molecules in a 'heterogeneous sea' of proteins, fibres, etc. The non-ambiguous answer of the assay is ensured by the specific reaction between antibody and antigen. With homogeneous assays, it is possible to detect proteins in the picomolar range in blood serum without washing and

\footnotetext{
* Copyright 2006 Society of photo-Optical Instrumentation Engineers.

This paper was published in Proceedings of SPIE Vol. 6191, pp. 234-241 (2006) and is made available as an electronic reprint with permission of SPIE. One print or electronic copy may be made for personal use only. Systematic or multiple reproduction, distribution to multiple locations via electronic or other means, duplication of any material in this paper for a fee or for commercial purposes, or modification of the content of the paper are prohibited.

${ }^{\dagger}$ FIA, referring to fluorescence in immunoassays, is commonly used throughout the literature. We prefer the term luminescence as it is a more general notion for radiative transitions.
} 
separating steps. This is achieved by the 'sandwich' architecture, with two antibodies bound to the same target antigen (see Figure 1A). The donor luminophore of one antibody is excited by laser light. FRET towards the acceptor luminophore of the second antibody only occurs when both antibodies are bound together via the antigen. Thus, the emission of the first luminophore is quenched while the latter is then able to luminesce, working as a measure for the antigen concentration.

The donor comprises an $\mathrm{Eu}^{3+}$ ion surrounded by the so called cryptand trisbipyridin (TBP) resulting in the cryptate EuTBP. ${ }^{5,6}$ Due to an internal energy transfer from the cryptand to the lanthanide, a spectral shift of about $300 \mathrm{~nm}$ is achieved between absorption and luminescence. The $\mathrm{Eu}^{3+}$ luminesces with a long decay time (hundreds of microseconds). This allows delay of the detection until the short lived autofluorescence from the surrounding matrix has faded away. Then only the photons from the donors and the bound acceptors are seen. Calculating the ratio of bound and non-bound antibodies provides the concentration of antigen. This technique is already applied in commercially available systems (such as Kryptor ${ }^{\mathrm{TM}}$, Cezanne S.A., Nîmes, France).

A)

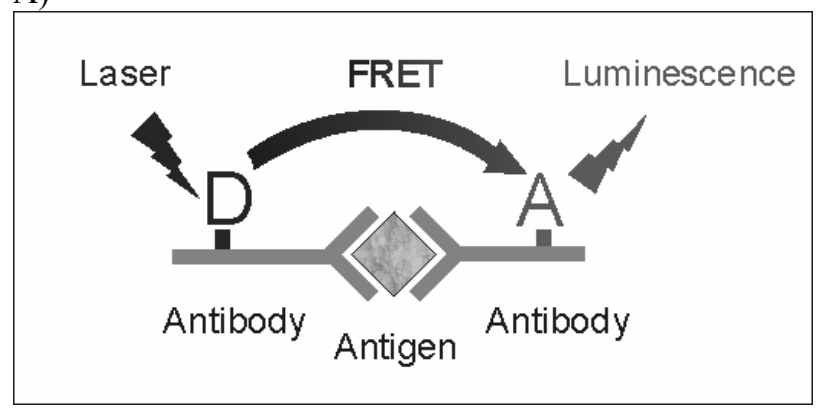

B)

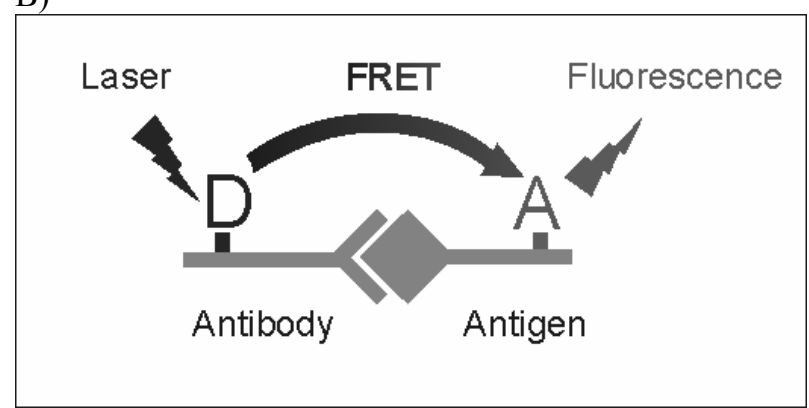

Figure 1 A) Scheme of Förster Resonance Energy Transfer (FRET) in an immunoassay. The antibody forms a 'sandwich' complex with the searched for antigen. The donor D absorbs the laser light and transfers it to the acceptor A nearby, which luminesces instead of the donor. B) Scheme of FRET in the test-assay. The donor attached to the antibody and the acceptor attached to the antigen are directly coupled.

In this work we demonstrate energy transfer from Eu-TBP donors to QD655 acceptors. The experiments were performed in solutions with and without serum to evaluate the influence on the FRET. ${ }^{7}$ After the excitation of Eu-TBP with a $\mathrm{N}_{2}$-laser and as result of the energy transfer, the luminescence of the QD655 acceptors also showed increased decay times. Instead of the whole immunoassay (Figure 1A), a simpler test scheme (Figure 1B) was used: the Eu-TBP donors were conjugated with streptavidin (Eu-TBP-Strep) and could directly couple to the QD655 acceptors conjugated with biotin (QD655-Biot) (see Figure 2). The intensities and the decay times were measured. The FRET efficiency, $\eta_{\text {FRET }}$, is the efficiency of the energy transfer in the coupled FRET pair and was calculated by: ${ }^{8}$

$\eta_{\text {FRET }}=1-\tau_{\mathrm{DA}} / \tau_{\mathrm{D}}$

Here, $\tau_{\mathrm{D}}$ and $\tau_{\mathrm{DA}}$ are the decay times for the uncoupled donor and for the donor coupled to the acceptor.

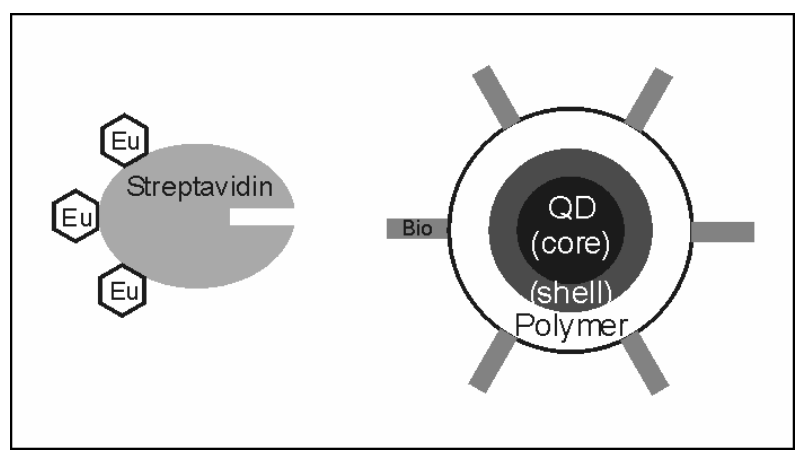

Figure 2 Eu-TBP coupled to streptavidin (Eu-TBP-Strep) is able to form FRET pairs with polymer-coated core-shell QDs which are marked with biotin (QD655-Biot). Streptavidin forms a very tight binding with biotin. In the experiments, ca. 3.1 Eu-TBPs are conjugated with one streptavidin and ca. 6 biotins with one QD655. 


\section{MATERiAls AND METHODS}

The FRET donor molecule is the cryptate $\mathrm{Eu}^{3+}$-trisbipyridin (Eu-TBP). For the binding assays, it is coupled to streptavidin (Eu-TBP-Strep). For the non-binding assays, the cryptate was coupled to tPSA (total prostate specific antigen). To improve the luminescence of Eu-TBP-Strep, KF was added resulting in a final concentration of $0.4 \mathrm{M}$ in the assays. The serum assays contained 33\% bovine serum. All cryptates, KF and serum were provided by Cezanne SA (Nîmes, France). The FRET acceptors were CdSe-ZnS core-shell quantum dots emitting at $655 \mathrm{~nm}$ (QD655), coupled to biotin (QD655-Biot) with a conjugating ratio biotin/ $\mathrm{QD}=6$. They were supplied by Quantum Dot Corp. (Hayward, CA, USA). The concentration series was measured with varying concentrations of Eu-TBP-Strep between $0 \mathrm{M}$ and $3.0 \cdot 10^{-8} \mathrm{M}$ which results in concentrations of streptavidin up to $9.6 \cdot 10^{-9} \mathrm{M}$ (i.e. a conjugating ratio Eu-TBP/Strep $=3.1$ ) and constant QD655-Biot concentrations of $4.0 \cdot 10^{-10} \mathrm{M}$ (biotin concentration $2.4 \cdot 10^{-9} \mathrm{M}$ ). This yields concentration ratios $c($ Strep $) / c($ Biot $)$ between 0 and 4 .

The FRET measurements were done with a commercial FIA reader system (KRYPTOR ${ }^{\mathrm{TM}}$, Cezanne SA, Nîmes, France) utilizing two single photon counting channels at $655 \mathrm{~nm}$ (channel A, acceptor signal, QD channel) and $620 \mathrm{~nm}$ (channel B, donor signal, $\mathrm{Eu}^{3+}$ channel) with $2 \mu$ s integration steps over $8 \mathrm{~ms}$ (see Figure 3). For the spectrally resolved measurements, a spectrometer was coupled with an ICCD camera (iStar DH720, Andor Technology, Belfast, Northern Ireland). The spectra were taken with a $200 \mu$ s delay from the laser pulse and with a measurement window of $1000 \mu \mathrm{s}$. The signal was integrated over 40 pulses. The light source in both cases was a $\mathrm{N}_{2}$-laser emitting at $337 \mathrm{~nm}$ with a $20 \mathrm{~Hz}$ repetition rate.

The data were fitted by a two-step global fitting procedure using a set of monoexponential decay functions: first, the decay time of Eu-TBP-Strep without acceptors was determined, then, the signals of all concentration measurements at both channels were fitted with this decay time and a global second decay time for the FRET-signal. This avoids falsely enlarged FRET decay times for the weak signals when a strong signal of uncoupled Eu-TBP-Strep is present. Thus for each concentration one gets a fourfold set of amplitudes $A_{i}$ and decay times $\tau_{i}$ : two pairs for the luminescence contribution of uncoupled Eu-TBP-Strep in both channels and two pairs for the FRET signal in both channels as the donor (at $620 \mathrm{~nm}$ ) and the acceptor (at $655 \mathrm{~nm}$ ) both take part in the FRET decay (see Figure 4). From these decay times and amplitudes the intensity of the contributions were calculated by:

$I_{i}=\int_{0}^{\infty} A_{i} \cdot \exp \left(-t_{i} / \tau_{i}\right) d t_{i}=A_{i} \cdot t_{i}$

As Eu-TBP emits with $1000 \mu$ s decay times and the FRET signal with decay times over $600 \mu$ s, the total intensity can be calculated from the delayed signal, omitting the intense short-time autofluorescence by neglecting the first $200 \mu \mathrm{s}$.

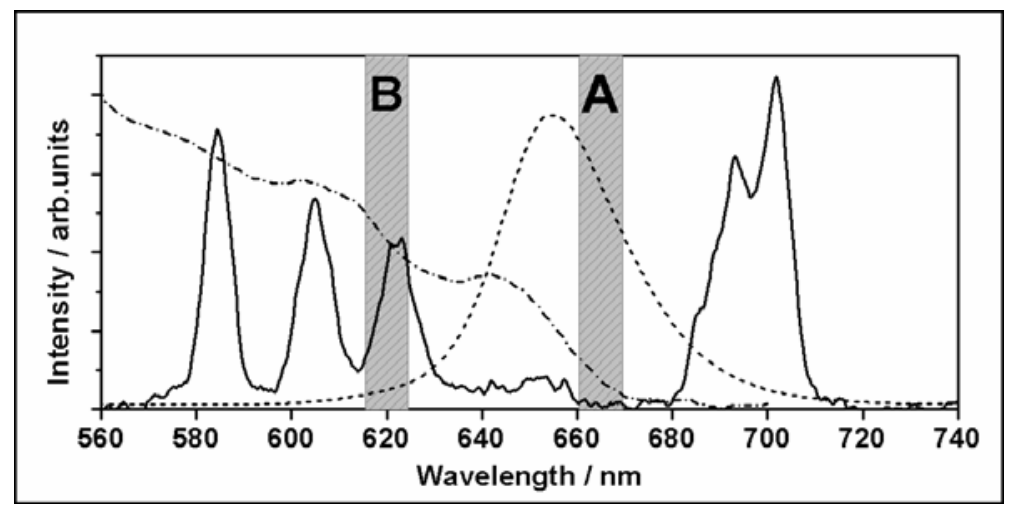

Figure 3 Emission spectra of donor Eu-TBP-Strep (solid line) and acceptor QD655-Biot (dotted line). The excitation spectrum of QD655-Biot is also included (dash-dotted line). The grey bars indicate the spectral detection regimes of the acceptor channel A $(665 \mathrm{~nm})$ and the donor channel B $(620 \mathrm{~nm})$. 
A)

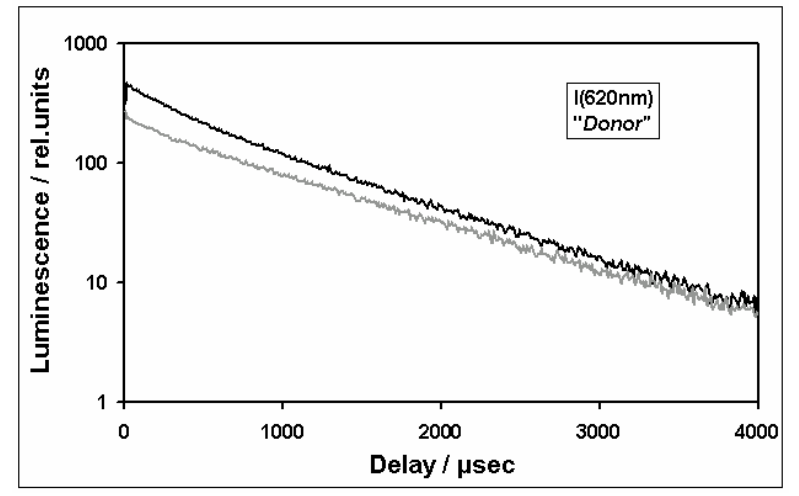

B)

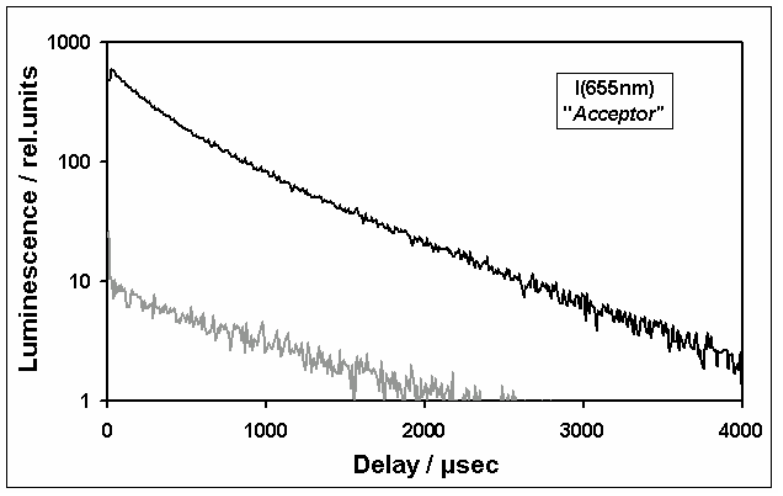

Figure 4 Decay measurements of pure donor (grey) and donor-acceptor mixtures (black) A) in the $620 \mathrm{~nm}$ channel and B) in the $665 \mathrm{~nm}$ channel $\left(c(\mathrm{QD} 655-\mathrm{Biot})=4.0 \cdot 10^{-10} \mathrm{M} ; c(\right.$ Eu-TBP-Strep $)=3.0 \cdot 10^{-8} \mathrm{M}$; no serum $)$. The intense FRET signal can be detected over the whole long-time range. The curves can be analyzed as biexponential decays $\left(\tau_{\mathrm{Eu}} \approx 1000 \mu \mathrm{s}\right.$ and $\left.600 \mu \mathrm{s}<\tau_{\mathrm{FRET}}<800 \mu \mathrm{s}\right)$.

\section{RESUlts AND Discussion}

The spectra of the assays containing Eu-TBP-Strep and that with the Eu-TBP-Strep/QD655-Biot mixture of show significant differences in the spectral region around $665 \mathrm{~nm}$ (see Figure 5). This means that the QD655-Biots, which usually have a short-time emission, show now long-time luminescence, which is clearly detectable after $200 \mu$ s. This is due to an energy transfer from the excited Eu-TBP to the QD655 if they are coupled via streptavidin and biotin. No difference can be seen between the emission spectra of the uncoupling Eu-tPSA/QD655-Biot mixture and the solutions without acceptors in the long-time range as the luminescence decay of QD655-Biot is too fast for this detection window. Therefore, a diffusion enhanced energy transfer can be excluded for the used concentrations. An energy transfer is only detectable between coupled FRET partners. No spectral displacement of bands were detected between assays with and without serum.

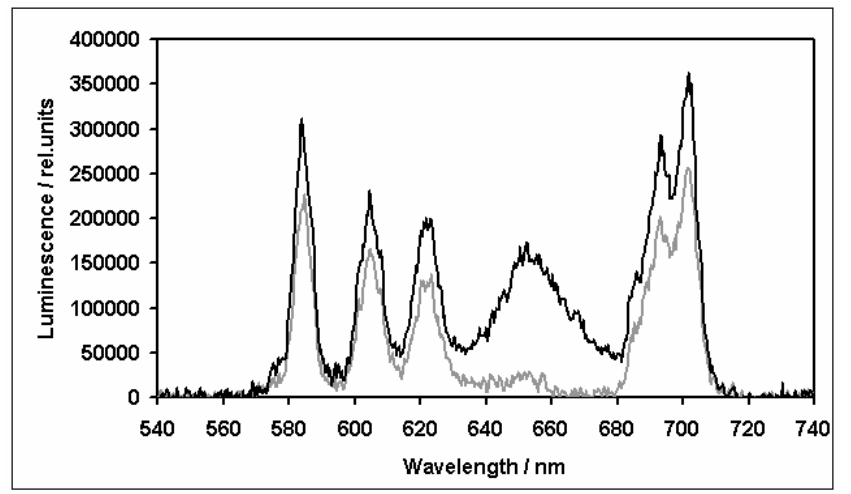

Figure 5 Emission spectra of Eu-TBP-Strep $\left(c=1.6 \cdot 10^{-9} \mathrm{M}\right.$; grey line) and of the Eu-TBP-Strep/QD655-Biot mixture (black line) $c($ Eu-TBP-Strep $)=1.6 \cdot 10^{-9} \mathrm{M} ; c(\mathrm{QD} 655$-Biot $\left.)=2.3 \cdot 10^{-9} \mathrm{M}\right)$. Both assays contained serum. The detection window had a delay of $200 \mu \mathrm{s}$ and a duration of $1000 \mu \mathrm{s}$.

The FRET luminescence intensity (channel A) of the serum-free FRET assay rises with increasing concentration of EuTBP-Strep until it gets saturated at $c($ Strep $) / c($ Biot $) \approx 3$ (see Figure 6 ). The signal of the serum containing assay shows the same tendency with lower intensities ending at $60 \%$ of the saturation intensity of the serum-free assay. The onset of 
saturation at higher concentration in the serum solutions indicates the unspecific binding concurring with the specific binding of streptavidin with biotin has previously been described. ${ }^{7}$

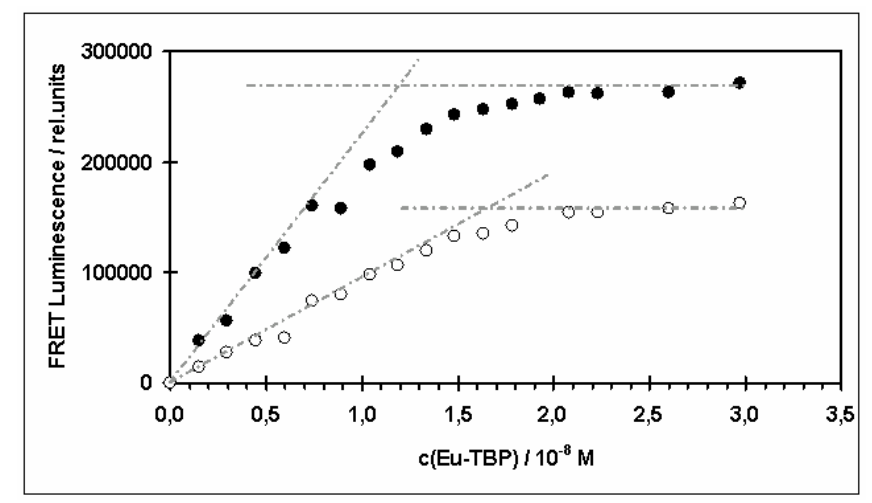

Figure 6 FRET luminescence (channel A; $665 \mathrm{~nm}$ ) at different Eu-TBP-Strep concentrations $\left(c(\right.$ QD655-Biot $\left.)=4.0 \cdot 10^{-10} \mathrm{M}\right)$. Full circles indicate signals in assay without serum, open circles in serum assays. The dark dash-dot lines are just to guide the eye.

The Eu-TBP-Strep luminescence intensity (channel B) shows a similar behaviour: it rises with increasing Eu-TBP-Strep concentration (see Figure 7$)$. Until $c($ Strep $) / c(B i o t) \approx 1,2$, the slope is steeper than at higher ratios when the intensity grows with the same slope as that from acceptor-free Eu-TBP-Strep. The difference between the Eu-TBP-Strep luminescence in the FRET assay and that of the pure Eu-TBP-Strep solution shows that the additional increase behaves like the FRET signal (channel A). The gain in luminescence is saturated at about the same Eu-TBP-Strep concentrations as in channel A. This means that there is not only an increase due to growing Eu-TBP-Strep concentration but also an increase in intensity due to the presence of the QD655-Biots. 'Crosstalk' luminescence can be excluded from the spectra as the luminescence of $\mathrm{Eu}^{3+}$ is equally enhanced in all bands (see Figure 5). This means that the gain is not due to luminescence from the FRET induced QD band centred at $655 \mathrm{~nm}$ 'shining' into channel B (Eu ${ }^{3+}$ channel; $\left.620 \mathrm{~nm}\right)$.

A)

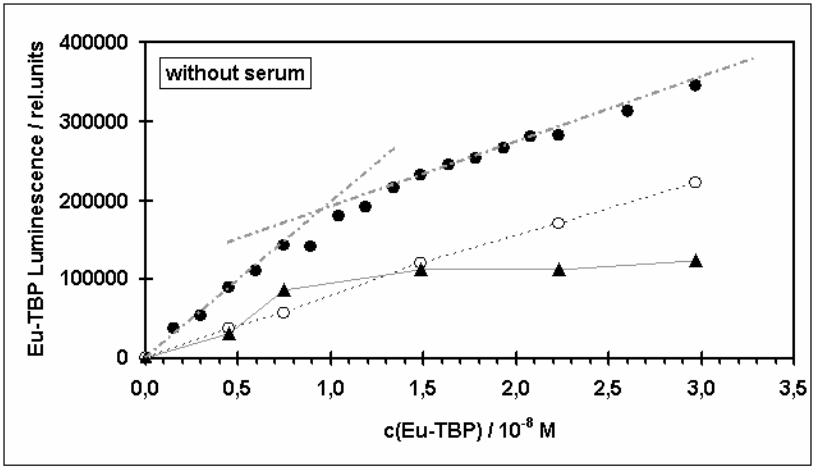

B)

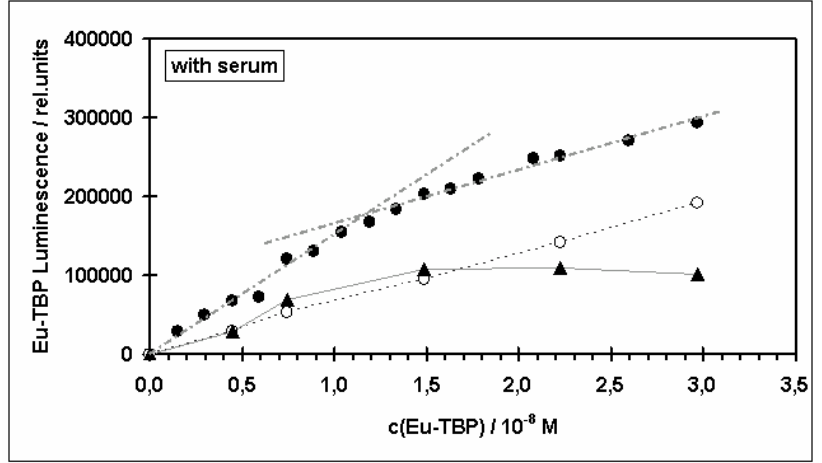

Figure 7 Eu-TBP-Strep luminescence (channel B) measured at different Eu-TBP-Strep concentrations. Full circles indicate signals in FRET assay $\left(c(\mathrm{QD} 655-\mathrm{Biot})=4.0 \cdot 10^{-10} \mathrm{M}\right)$, open circles in acceptor-free assays and triangles the difference between both. The grey dash-dot lines are to guide the eye. A) Assay without serum. B) Assay with serum.

A look at the decay times evaluated from the measurements with concentration dependence gives a more detailed picture (see Table 1): the FRET decay times in solutions with and without serum differ significantly by $13 \%$. This results in a maximum FRET efficiency of 37\% from Eu-TBP-Strep to QD655-Biot (serum free assays) whereas the FRET efficiency in the serum solution is $27 \%$. Thus, the serum reduces the FRET efficiency by ca. one third. This means that the serum does not only influence the availability of free binding places allowing FRET pairs, it also influences the properties of the energy transfer. 


\begin{tabular}{|l|c|c|c|c|}
\hline Assays $\ldots$ & $\tau_{\text {Eu }}$ & $\tau_{\text {FRET }}$ & $\eta_{\text {FRET }}$ & $R$ \\
\hline without serum & $1080 \mu \mathrm{s} \pm 40 \mu \mathrm{s}$ & $690 \mu \mathrm{s} \pm 50 \mu \mathrm{s}$ & $0.36 \pm 0.02$ & $85 \AA \pm 1 \AA$ \\
\hline with serum & $1060 \mu \mathrm{s} \pm 30 \mu \mathrm{s}$ & $790 \mu \mathrm{s} \pm 40 \mu \mathrm{s}$ & $0.25 \pm 0.04$ & $91 \AA \pm 3 \AA$ \\
\hline
\end{tabular}

Table 1 Luminescence decay times of $\mathrm{Eu}^{3+}\left(\tau_{\mathrm{EU}}\right)$ and FRET $\left(\tau_{\text {FRET }}\right)$ and the resulting FRET-efficiencies $\left(\eta_{\text {FRET }}\right)$ with their maximum errors. The distances $R$ between donor and acceptor were calculated with a Förster-radius of $77 \AA$ (see text).

The FRET efficiency is connected with the distance $R$ between donor and acceptor by the Förster distance $R_{0}:{ }^{8}$

$\eta_{\text {FRET }}=\frac{R_{0}^{6}}{R_{0}^{6}+R^{6}}$

The Förster distance $R_{0}$ of the Eu-TBP-Strep and QD655-Biot pair was calculated from the overlap integral $J$ of the donor emission band and acceptor absorption band (see Figure 3):

$R_{0}=\left(8.79 \cdot 10^{-5} \cdot Q_{\mathrm{D}} \kappa^{2} n^{-4}\right)^{1 / 6} J^{1 / 6}$ in $\AA$

with the donor luminescence efficiency $Q_{\mathrm{D}}$, the orientation factor $\kappa^{2}$ and the refraction index $n$. $J$ was calculated to $6.2 \cdot 10^{16} \mathrm{M}^{-1} \mathrm{~cm}^{-1} \mathrm{~nm}^{4.8}$. The donor luminescence efficiency is that of the $\mathrm{Eu}^{3+}$ in the cryptate $Q_{\mathrm{D}}=Q_{\mathrm{Eu}}=0.2$, not of the whole cryptate Eu-TBP-Strep $\left(Q_{\mathrm{D}}=0.02\right){ }^{9}{ }^{910}$ With $\kappa^{2}=2 / 3$ (isotropic orientation) and $n=1.4$ (biomolecules in aqueous solutions) ${ }^{11}$ the Förster distance amounts to $77 \AA$. According Equations (3) and (4), one reason for a change of $\eta_{\text {FRET }}$ is a different distance $R$. Due to the same donor decay times $\tau_{\mathrm{D}}$, no change in the quantum efficiency of the donor $Q_{\mathrm{D}}$ can be expected. The orientation of the molecules are possibly influenced by the additional molecules. The refractive index does not change for the biomolecule concentrations considered. ${ }^{11}$

The binding efficiency $\eta_{\text {Bind }}$ can be calculated from the different decay contributions and is defined here as the numbers of bound donors $n_{\mathrm{b}}$ divided by the sum of bound and unbound donors $n_{\mathrm{b}}+n_{\mathrm{nb}}$. If FRET is the only distinguishing property between $n_{\mathrm{b}}$ and $n_{\mathrm{nb}}$ the assay efficiency can be calculated according to:

$\eta_{\text {Bind }}=\frac{n_{\mathrm{b}}}{n_{\mathrm{b}}+n_{\mathrm{nb}}}=\frac{I_{\mathrm{DA}} /\left[Q_{\mathrm{Eu}}\left(1-\eta_{\mathrm{FRET}}\right)\right]}{I_{\mathrm{D}} / Q_{\mathrm{Eu}}+I_{\mathrm{DA}} /\left[Q_{\mathrm{Eu}}\left(1-\eta_{\mathrm{FRET}}\right)\right]}=\frac{I_{\mathrm{DA}}}{I_{\mathrm{D}}\left(1-\eta_{\mathrm{FRET}}\right)+I_{\mathrm{DA}}}$

The intensities $I_{\mathrm{D}}$ and $I_{\mathrm{DA}}$ are the intensities of the uncoupled donor and of the donor coupled to an acceptor molecule respectively. This can be understood as the absorbed energy in the bound molecules divided by sum of absorbed energy in the bound and the unbound molecules. The term $I_{\mathrm{DA}} /\left(1-\eta_{\mathrm{FRET}}\right)$ is the non-transferred emitted light of the coupled donors divided by the 'non-transfer efficiency'. Thus, the binding efficiency can be calculated using only the luminescence contributions of $\mathrm{Eu}^{3+}$ (channel B).

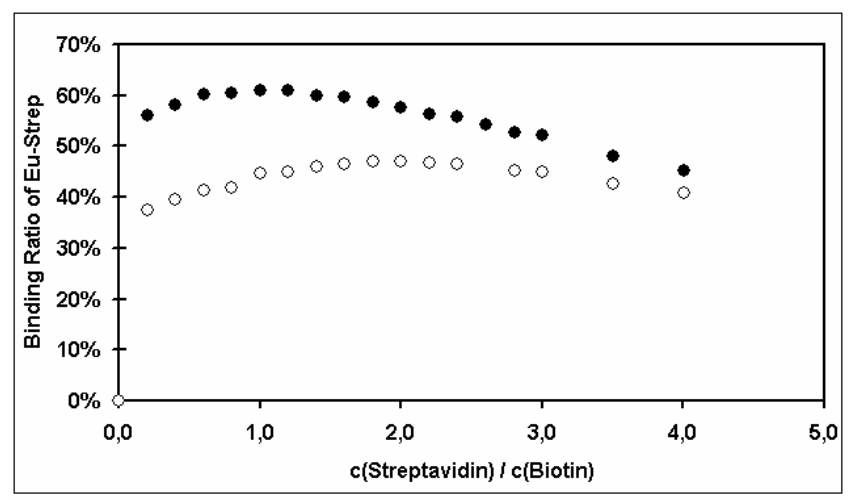

Figure 8 Binding ratio of Eu-TBP-Strep over the concentration ratio. Full circles indicate binding ratios in serum free samples, open circles in serum samples.

The maximum binding ratio is $60 \%$ at $c($ Strep $) / c($ Biot $) \approx 1$ for the serum free assay and $47 \%$ at $c($ Strep $) / c($ Biot $) \approx 2$ for the serum containing assay (see Figure 8). With growing excess of Eu-TBP-Strep the binding efficiency decreases. Serum promotes unspecific binding, such reducing the overall FRET efficiency of the assay. 
The origin of the gain in luminescence in channel B can be found looking at the intensities of the uncoupled Eu-TBPStrep. The intensity of the unbound Eu-TBP-Strep in acceptor free assays and in QD655-Biot containing assays is shown in Figure 9. One can see that the luminescence of the free Eu-TBP-Strep is not reduced in FRET assays as one would expect but has the same intensity. In serum assays, the Eu-TBP-Strep luminescence is even higher in the presence of QD655-Biot (not shown). This shows that in the presence of the QD655-Biot acceptors, the Eu-TBP-Strep luminescence is enhanced. The reason for this enhancement is subject of ongoing research.

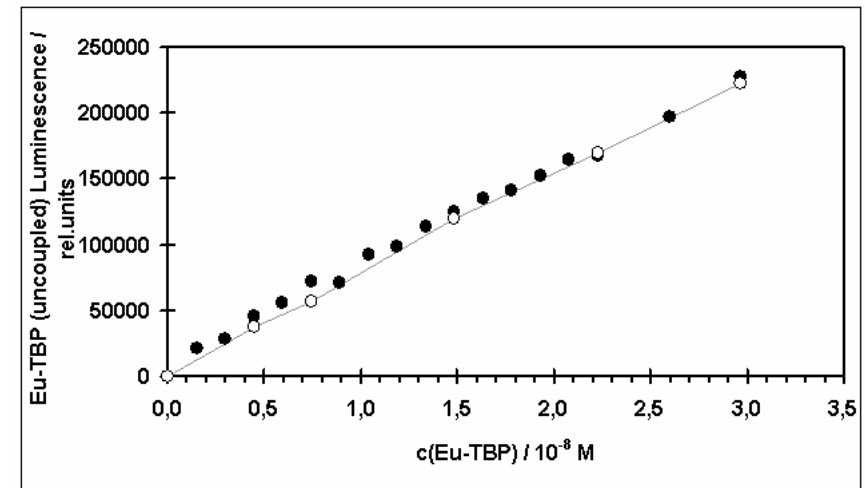

Figure 9 Luminescence of the uncoupled Eu-TBP-Strep (these are the components at $620 \mathrm{~nm}$ with decay times at about $1000 \mu \mathrm{s}$ ) at different Eu-TBP-Strep concentrations. Full circles indicate signals in FRET assays $\left(c(\right.$ QD655-Biot $\left.)=4.0 \cdot 10^{-10} \mathrm{M}\right)$, open circles in acceptor free assays. (All assays were serum-free.)

\section{ConClusion}

We have shown FRET between Eu-TBP and QD655, if they are coupled by use of streptavidin and biotin. The FRET signal is intense in the measurement window thus enabling good signal-to-noise ratios not only for the Eu-TBP-Strep donor signal bat also for the FRET signal. Using serum assays, the FRET signal is reduced in comparison to serum free assays. The reason for this decrease is twofold: the unspecific binding of serum molecules occupies coupling sites of the FRET partners, and the serum reduces the FRET efficiency probably due to steric hindering effects which separates the FRET partners further than without serum. The conditions for this effect should be further investigated in order to get reliable assays with QDs as acceptors. Nevertheless, QDs are very promising substitutes for organic acceptors in FRET as their stability and their narrow emission bands provide the chance for new immunoassays, e. g. enabling multiplexing.

\section{ACKNOWLEDGEMENT}

This work was supported by the German Bundesministerium für Wirtschaft und Arbeit (InnoNet 16IN0225).

\section{REFERENCES}

1 a) D.M. Willard, L.L. Carillo, J. Jung, A.V. Orden, "CdSe-ZnS Quantum Dots as Resonance Energy Transfer Donors in a Model Protein-Protein Binding Assay.”, Nano Lett., 1, 469-74, 2001.

b) N. Mamedova, N. A. Kotov, A. L. Rogach and J. Studer, "Albumin-CdTe Nanoparticle Bioconjugates: Preparation, Structure, and Interunit Energy Transfer with Antenna Effect", Nano Lett., 1, 281-286, 2001.

c) A. R. Clapp, I. L. Medintz, J. M. Mauro, B. R. Fisher, M. G. Bawendi and H. Mattoussi, "Fluorescence Resonance Energy Transfer Between Quantum Dot Donors and Dye-Labeled Protein Acceptors", J.Am.Chem.Soc., 126, 301-310, 2004.

d) E. Alphandéry, L. M. Walsh, Y. Rakovich, A. L. Bradley, J. F. Donegan and N. Gaponik, "Highly efficient Förster 
resonance energy transfer between CdTe nanocrystals and Rhodamine B in mixed solid films", Chem.Phys.Lett., 388, 100-104, 2004.

2 A.L. Clapp, I.L. Medintz, B.R. Fisher, G.P. Anderson, H. Mattoussi, „Can Luminescent Quantum Dots Be Efficient Energy Acceptors with Organic Dye Donors?”, J.Am.Chem.Soc., 127, 1242-1250, 2005.

${ }^{3}$ N.Hildebrandt, L.J. Charbonnière, M.Beck, R. Ziessel, H.-G. Löhmannsröben, „Quantum Dots as Efficient Energy Acceptors in a Time-Resolved Fluoroimmunoassay“, Angew.Chem.Int.Ed., 44, 7612-15, 2005.

${ }^{4} \mathrm{D}$ Wild (Ed.), The immunoassay handbook. $2^{\text {nd }}$ ed., Nature Publishing Group, London, 2001.

${ }^{5}$ G. Mathis, F. Socquet, M. Viguier and B. Darbouret, "Homogeneous Immunoassays Using Rare Earth Cryptates and Time Resolved Fluorescence: Principles and Specific Advantages for Tumor Markers" Anticancer Res., 17, 3011-3014, 1997.

${ }^{6}$ G. Mathis, "Rare Earth Cryptates and Homogeneous Fluoroimmunoassays with Sera" Clin.Chem., 38, 1953-1959, 1993.

${ }^{7}$ J. H. F. Erkens, S. J. Dieleman, R. A. Dressendörfer and C. J. Strasburger, "A time-resolved fluoroimmunoassay for cortisol in unextracted bovine plasma or serum with optimized procedures to eliminate steroid binding protein interference and to minimize non-specific streptavidin-europium binding" J.Steroid Biochem.Mol.Biol., 67, 153-161, 1998.

${ }^{8}$ T. Förster, "Zwischenmolekulare Energiewanderung und Fluoreszenz" Ann.Phys., 2, 55-75, 1948. Cited in B.W. van der Meer, G. Coker, III, S.-Y.S. Chen, Resonance Energy Transfer: Theory and Data, VCH Verlags GmbH, Weinheim, 1994.

${ }^{9}$ B. Alpha, R. Ballardini, V. Balzani, J.-M. Lehn, S. Perathoner and N. Sabbatini, "Antenna Effects in Luminescent Lanthanide Cryptates: A Photophysical Study" Photochem. Photobiol., 52, 299-306, 1990.

${ }^{10}$ M.Xiao and Paul R. Selvin, "Quantum Yields of Luminescent Lanthanide Chelates and Far-Red Dyes Measured by Resonance Energy Transfer”, J.Am.Chem.Soc., 123, 7067-7073, 2001

${ }^{11}$ J. R. Lakowicz, Principles of Fluorescence Spectroscopy, Kluwer Academic/Plenum, New York, 1999 